\title{
El sueño chino 中国梦
}

\author{
Editado por/Edited by: Eduardo Castro \\ Recibido/Received: 26/01/2014. Aceptado/Accepted: 15/05/2014 \\ Publicado en línea/Published on Web: 10/09/2014
}

\author{
Kiara Guerra \\ Universidad San Francisco de Quito, Colegio de Ciencias Sociales y Humanidades COCISOH \\ Diego de Robles y Vía Interoceánica, Quito, Ecuador \\ Correo electrónico: kiara.guerra@estud.usfq.edu.ec
}

\section{Resumen}

El flamante presidente de la República Popular China, Xi Jinping, ha mencionado en más de una ocasión 'el sueño chino', haciendo referencia a un ideal que busca promover tanto a nivel nacional como internacional, en función de los recientes cambios en la política interna del gigante asiático y de su creciente influencia en todo el mundo. ¿De qué se trata el ‘sueño chino’? ¿Es el ‘sueño chino' el nuevo ‘sueño americano'? ¿Podría beneficiarse el Ecuador en el proceso de rejuvenecimiento de China a través del 'sueño chino'?

Palabras clave: Sueño chino, Xi Jinping, identidad socialista, desarrollo, Ecuador.

\section{Abstract}

The new president of the People's Republic of China, Xi Jinping, has mentioned on more than one occasion 'Chinese Dream', referring to an ideal that seeks to be promoted at both national and international level, according to the recent changes in domestic policy the Asian giant and its growing influence throughout the world. What is the 'Chinese dream'? Is the 'Chinese dream' the new American dream? Could it benefit Ecuador in the process of rejuvenating China through the 'Chinese dream'?

Palabras clave: Chinese dream, Xi Jinping, socialist identity, development, Ecuador.

Las consignas del ex presidente chino Hu Jintao, en lo interno una sociedad armoniosa, en lo externo un desarrollo pacífico y la evolución del concepto científico de desarrollo, guiaron su gobierno durante los últimos diez años. Junto con las consignas que destacaron a los otros seis presidentes de China desde la fundación de la República Popular en 1949, han tomado un giro inesperado, el cual pone en evidencia la evolución de dichas consignas a lo largo del tiempo. El actual presidente Xi Jinping ha introducido el sueño chino como su consigna, pero ¿qué es el sueño chino? Y, ¿cuál será el posible impacto de la consecución del sueño chino para el Ecuador, América Latina y el Caribe?

Xi Jinping, quien asumió su mandato el 14 de marzo del año pasado, empezó su periodo con un discurso alentador que marcó la política y el enfoque de su gobierno. En su intervención, el presidente Xi se refirió por primera vez a 'el sueño chino' ( 中国梦). Desde entonces el conjunto del aparato gubernamental se ha encargado de comunicar y transmitir a los ciudadanos chinos, a través de grandes campañas de difusión, el significado del mismo.

En aquel discurso, Xi Jinping se dirigió a todo el pueblo con respecto al significado del sueño chino. Mencionó que se debe tener en cuenta la misión, que unidos como uno solo pueden formar una fuerza invencible de sabiduría y poder. Asimismo recalcó que "todos los ciudadanos chinos merecen oportunidades iguales para disfrutar de una vida próspera, para ver sus sueños volverse realidad y juntos beneficiarse del desarrollo del país." Es evidente que Xi apela al sentimiento de patriotismo de su pueblo, y es sin duda uno de los objetivos del sueño chino el reafirmar el rol de la unidad de los ciudadanos chinos como la fuerza de la nación misma que busca alcanzar un ideal, no sólo de un desarrollo económico sino también de un desarrollo integral del país.

Xi también dice, "debemos hacer esfuerzos persistentes, seguir hacia adelante con voluntad indomable, continuar empujando hacia la gran causa que es el socialismo con características chinas, y esforzarnos por alcanzar el sueño chino de la gran revitalización de nuestro pueblo." Xi menciona algunos elementos clave para entender mejor qué es el sueño chino. Uno de ellos es el de la igualdad de oportunidades, para que cada uno de los ciudadanos pueda tener una vida próspera. Por lo tanto se está refiriendo a que el sueño chino busca alcanzar el bienestar de la población china, donde las personas puedan lograr sus metas personales a la par que el país sigue un camino conjunto hacia el desarrollo.

Asimismo, Xi recalca la importancia de lograr aquel desarrollo y bienestar de los ciudadanos, dentro del marco ideológico del socialismo con características chinas. Finalmente, otro elemento fundamental sobre el sueño chino en la intervención del presidente $\mathrm{Xi}$, radica en la idea de unidad, la cual no desaparece, sino que más bien debe ser reforzada, pues es la forma de cumplir el sueño chino. 


\section{Repercusión global}

Por otro lado, las reacciones y los análisis de otros países no se han hecho esperar, e inmediatamente han surgido varias interpretaciones en occidente sobre el significado del 'sueño chino', y del enfoque que el gobierno de Xi está buscando desarrollar y alcanzar al margen de esta gran consigna. La primera reacción de muchos fue tratar de entender el sueño chino, teniendo en cuenta la similitud que salta a la vista entre éste y su par estadounidense, el sueño americano. Éste planteaba la idea de la consolidación en la sociedad de una clase media que tenga una estabilidad económica, donde todas sus necesidades estén satisfechas, y que cada individuo podía alcanzar.

Otros han sugerido que el sueño chino representa, desde cierto punto de vista, un intento del presidente Xi por mejorar el diálogo con los Estados Unidos, dada la similitud semántica de los dos lemas que ambos países profesan. Adicionalmente, se ha relacionado la introducción de 'el sueño chino' por parte del presidente Xi, dado que en octubre del 2012 Thomas Friedman, articulista del diario New York Times, publicara un artículo titulado "China necesita su propio sueño" (China needs its own dream). Friedman afirmaba que si 'el sueño chino' era similar al sueño americano, en el sentido en el que se potenciaría el surgimiento de una clase media basada en el consumo, otro planeta sería necesario. Por esta razón, Friedman invitaba al presidente $\mathrm{Xi}$ a presentar un nuevo sueño chino, que sea compatible con las expectativas de la población por alcanzar prosperidad de la mano de una China más sustentable. Ésta es una de las interpretaciones que se han surgido sobre 'el sueño chino' en muchos países de occidente.

Cabe recalcar que una de las diferencias fundamentales entre el sueño chino y el sueño americano está relacionada con el hecho de que el sueño chino no busca exclusivamente el desarrollo económico del individuo, sino más bien de toda la población china en su conjunto, con un enfoque integral. Es decir, que China logre alcanzar no sólo la constitución de una sociedad próspera, sino también una sociedad comprometida con el futuro de la República Popular, que encuentre el balance entre el desarrollo económico y las características del socialismo chino. El sueño chino es por lo tanto la consecución de este equilibrio, que propicie la revitalización del pueblo chino, de sus valores culturales, propiciando una estrategia de apertura y crecimiento económico, pero conservando el modelo socialista con sus particularidades, inherentes a la República Popular.

Adicionalmente, en diciembre del año pasado tuvo lugar en Shanghai el Diálogo Mundial sobre el Sueño Chino (World Dialogue on the Chinese Dream) con el objetivo de establecer un espacio en el que se pueda difundir este ideal, y a su vez compartir opiniones respecto del mismo. A este evento asistieron alrededor de veinte estudiosos de todo el mundo, quienes durante dos días participaron de esta iniciativa con un tinte más académico. Gustaff Geeraerts, director del Instituto de Estudios
Contemporáneos de China, de la Universidad Libre de Bruselas, es uno de los académicos que asistió a este evento, y afirma que desde una perspectiva más cercana él distingue tres otros sueños en el sueño chino. "Primero, se trata de poner en práctica la revitalización de China, permaneciendo leales a una rica herencia cultural y a su propia identidad socialista. Segundo, se trata de una China fuerte con el objetivo de plantearse una política exterior independiente y resuelta a seguir su propio camino, mientras que al mismo tiempo se incremente la cooperación mutua con otros países, para que juntos enfrenten retos globales y puedan trabajar para hacer su contribución al desarrollo de todo el mundo. Tercero, se trata de un orden mundial dónde los Estados sean iguales y puedan confiar unos en otros, para así alcanzar seguridad colectiva, manteniendo la diversidad de culturas y hacia una cooperación ganar-ganar que resulte en prosperidad mutua."

Considero que es importante pensar el sueño chino con respecto a los tres puntos señalados por Geeraerts, pues resume brillantemente mucho de lo que es el sueño chino. Asimismo uno de los puntos clave de la interpretación presentada por este académico, es la importancia de mantener la identidad socialista china a lo largo de la consecución de los objetivos del sueño chino como son el desarrollo y crecimiento económico, así como en la integración y activa participación de China en el mundo. Si bien la interpretación de este académico es clara y concisa, es fundamental resaltar que el ideal del sueño chino sigue desarrollándose, por lo cual limitarnos a buscar una definición precisa de éste no es factible.

Li Keqiang, el nuevo Primer Ministro chino ha mencionado que China tiene una meta presente: la de construirse como un país próspero, fuerte, participativo*, con una cultura avanzada, y sobre todo como un armonioso y moderno país socialista. Hacia mediados de este siglo espera que esta meta se cumpla, y así también el sueño chino de gran revitalización de China. Asimismo, el Primer Ministro ha afirmado que China demostrará sus exhaustivos esfuerzos hacia la consecución de estos objetivos, y por ende el desarrollo de China proveerá vastas oportunidades para el mundo entero y sus metas hacia el desarrollo.

En efecto, el desarrollo del gigante asiático se ha evidenciado en un incremento en la inversión en América Latina y el Caribe De acuerdo con Alicia Bárcena, Secretaria Ejecutiva de la Comisión Económica de las Naciones Unidas para América Latina y el Caribe (CEPAL), en 2010 China invirtió 14000 millones de dólares. China ha propuesto la creación del Foro de Cooperación China-América Latina y el Caribe, que se presenta como una oportunidad de diálogo que busca fomentar las relaciones multilaterales.

En el caso del Ecuador, el incremento de las inversiones chinas en los últimos años han convertido a la República Popular en el cuarto mayor socio comercial de nuestro país. Sin duda el desarrollo que va de la mano del ideal de la realización del sueño chi- 
no, puede tener un impacto positivo en las relaciones comerciales de nuestro país, por lo tanto el fortalecimiento de las mismas es esencial. Es así que se celebró, en Beijing, en enero del presente año, el foro bilateral "Ecuador Crece". A este evento asistieron empresarios chinos y ecuatorianos con el objetivo de consolidar un acercamiento con el gigante asiático, que pueda llevar a la mejora de las relaciones comerciales entre ambos países.

Si bien es cierto el sueño chino plantea grandes retos para ese país a nivel interno y externo, también está definiendo algunos para América Latina y el Caribe, y por ende para el Ecuador. El mayor reto es definitivamente que la región vea un crecimiento económico representativo, pero ¿cuál es el precio de tan deseado desarrollo económico? Me uno a las palabras de Alicia Bárcena, el desarrollo que resulte, tanto para China como para nuestra región, del acercamiento en las relaciones comerciales entre ambos actores, debe ser pensado en función de la sostenibilidad del mismo. Evidentemente las oportunidades que presenta el sueño chino para otros países en desarrollo, pueden seguir teniendo un impacto positivo en nuestras economías, o bien pueden tomar un giro tal vez inesperado, pero conocido $\mathrm{y}$ recorrido por América Latina y el Caribe en décadas pasadas.

El sueño chino es un ideal que busca alcanzar la revitalización del pueblo chino, junto con un crecimiento económico que propicie el bienestar de la población, y que mantenga a lo largo de este proceso de implementación de políticas públicas las características del socialismo chino. Es decir, el sueño chino se plantea por lo pronto como un equilibrio entre aquella identidad y tradición socialista en la República Popular, y un crecimiento económico que logre el bienestar de una población, que a la par mantenga una armonía con sus valores culturales. Sin duda el sueño chino representa un gran reto, no sólo para la administración de $\mathrm{Xi}$ a cargo de poner en práctica políticas públicas que logren evidenciar el proceso hacia la consecución de este ideal, sino que también representa un reto para el Partido Comunista Chino y para la población en sí para que sean partícipes y partidarios de los cambios que el sueño chino pueda traer para la República Popular.

* La palabra original utilizada por la fuente es "democrático", sin embargo he decidido cambiarla por "participativo", pues no me ha sido posible esclarecer si la traducción del chino al español es la correcta, o la mejor. Cabe recalcar que la concepción sobre participación o democracia en la República Popular China debe variar sustancialmente de la nuestra, explicar esta gran diferencia requeriría de un profundo análisis en el desarrollo de otro artículo.

\section{Referencias}

Baijie, A., Zhu, J., \& Xinhua. (18 de Marzo de 2013). 'Chinese dream 'is Xi's vision. Recuperado de China Daily: http:// cpcchina.chinadaily.com.cn/2013-03/18/content_16316325. htm

Chasing the Chinese dream. (2 de Mayo de 2013). Recuperado el 2014, de The Economist: http:/www.economist. com/news/briefing/21577063-chinas-new-leader-has-beenquick-consolidate-his-power-what-does-he-now-want-his

Geeraerts, G. (8 de Diciembre de 2013). Seminar on Chinese Dream: a dream shared by the world. Recuperado de China. org.cn: http://www.china.org.cn/china/Chinese_dream_dialogue/2013-12/08/content_30887410_4.htm

Huan, Y., \& Gang, D. (2 de diciembre de 2013). El "Sueño Chino"contribuye al "Sueño Latinoamericano". Recuperado de Pueblo en Línea: http://spanish.people.com. $\mathrm{cn} / 31619 / 8472533 . h t m l$

Profile: Xi Jinping. (5 de Junio de 2013). Recuperado de BBC: http://www.bbc.com/news/world-asia-pacific-11551399

Xinhua. (29 de mayo de 2013). China's economic growth offers opportunity: Li. Recuperado de China Daily: http://cpcchina.chinadaily.com.cn/2013-04/29/content_16469028.htm

Xinhua. (17 de Marzo de 2013). Xi vows to press ahead with 'Chinese dream'. Recuperado de China Daily: http://cpcchina.chinadaily.com.cn/2013-03/17/content_16316321.htm

Xinhua. (24 de Enero de 2014). ENTREVISTA: Relación China-Ecuador en mejor momento histórico, dice vicepresidente ecuatoriano. Recuperado de Xinhua News: http://spanish. xinhuanet.com/chinaiber/2014-01/24/c_133070874.htm 